\title{
Flagellin promotes the proliferation of gastric cancer cells via the Toll-like receptor 5
}

\author{
EUN-JUNG SONG ${ }^{1}$, MIN-JUNG KANG ${ }^{1}$, YONG-SEOK KIM ${ }^{2}$, SUN-MOON KIM ${ }^{2}$, \\ SANG-EOK LEE ${ }^{3}$, CHANG-HWAN KIM ${ }^{4}$, DONG-JAE KIM ${ }^{5}$ and JONG-HWAN PARK ${ }^{1}$
}

\begin{abstract}
Departments of ${ }^{1}$ Biochemistry, ${ }^{2}$ Internal Medicine and ${ }^{3}$ Surgery, College of Medicine, Konyang University, Daejeon 302-711; ${ }^{4}$ Agency for Defense Development, Daejeon 305-152, Republic of Korea; ${ }^{5}$ Department of Pathology, University of Michigan Medical School, Ann Arbor, MI 48109, USA
\end{abstract}

Received January 19, 2011; Accepted March 3, 2011

DOI: $10.3892 / \mathrm{ijmm} .2011 .656$

\begin{abstract}
Signaling of the Toll-like receptor (TLR) is closely associated with tumor development and progression processes including cell proliferation, angiogenesis, metastasis, and immunosuppression. In this study, we examined the expression of TLR5 in gastric cancer cells and its function in cell proliferation. RT-PCR revealed that the TLR5 gene was expressed in all gastric cancer cell lines examined, SNU638, SNU601, SNU216, and AGS. The TLR5 agonist, flagellin, induced IL-8 production and NF- $\mathrm{KB}$ activation in the gastric cancer cell lines. In addition, flagellin enhanced the proliferation of all gastric cancer cells examined, whereas LPS did not affect that of SNU638 cells. Blockade of TLR5 using an antibody, restored the proliferation of SNU638 cells enhanced by flagellin, indicating that TLR5 is essential for cell proliferation by flagellin. Flagellin also led to phosphorylation of ERK in SNU638 cells. The ERK inhibitor, PD98059, restored the proliferation ability of SNU638 cells enhanced by flagellin, suggesting that ERK may play an important role in the proliferation of gastric cancer cells. These findings suggest that TLR5 may play an important role in tumor progression of gastric cancer via the regulation of cell proliferation.
\end{abstract}

\section{Introduction}

Pattern recognition receptors (PRRs) are a series of innate immune receptors including Toll-like receptors (TLRs), Nod-like receptors (NLRs), and an RNA helicase family of receptors. Sensing of pathogen-associated molecular patterns (PAMPs) by PRRs is critical for initiation of the inflammatory response. TLRs are type I transmembrane receptors which

Correspondence to: Dr Jong-Hwan Park, Department of Biochemistry, College of Medicine, Konyang University, 685 Gasuwon-dong, Seo-gu, Daejeon 302-711, Republic of Korea

E-mail: jonpark@konyang.ac.kr

Key words: flagellin, gastric cancer, proliferation, Toll-like receptor, extracellular-signal regulated kinase are structurally characterized by extracellular leucine rich repeats (LRRs) and an intracellular Toll/IL-1R (TIR) domain. Extracellular surface TLRs including TLR2, TLR4, and TLR5 recognize bacterial lipoproteins, lipopolysaccharide (LPS), and flagellin, respectively (1). On the other hand, endosomal TLRs are composed of TLR3, TLR7/8, and TLR9 and are responsible for sensing nucleic acids such as dsRNA (TLR3), ssRNA (TLR7/8), and the CpG motif from bacterial and viral DNA (TLR9) (1). In addition to microbial molecules, a variety of endogenous ligands such as necrotic or dying cells, heat shock proteins, high mobility group box 1 (HMGB1), hyaluronan fragments, heparin sulphate, and fibronectin are recognized by TLR2 or TLR4 (2). TLR activation leads to the activation of NF- $\mathrm{BB}$ or interferon regulatory factors (IRFs) through the MyD88-dependent and/or MyD88-independent (TRIF-dependent) pathway and subsequently produce proinflammatory cytokines or type I interferons $(1,3)$.

TLRs are also known to be closely associated with tumor development $(4,5)$. Many studies have shown that TLR activation promotes tumorigenesis in a variety of carcinomas by enhancing cell proliferation, angiogenesis, and immunosuppression (6-13). In addition, TLRs mediate migration or invasion of several tumors, which are critical processes for cancer metastasis (7,14-16).

Gastric adenocarcinoma is a leading cause of cancerrelated death in the world, ranking as the second cause in males and the fourth in females (17). Over the world, more than 700,000 people per year die due to this malignancy (18). Helicobacter pylori infection is the best-known risk factor for gastric adenocarcinoma, and etiological studies have revealed that the attributable risk for gastric cancers conferred by $H$. pylori is approximately $75 \%$ (19). There is evidence supporting the relation between TLR4 polymorphism and the risk of gastric cancers $(20,21)$. It has also been reported that $H$. pylori augments the growth of gastric cancers via TLR2 or TLR4 pathways $(22,23)$. These findings suggest that TLR signaling may play an important role in the development and progression of gastric cancer. A clinical study has shown that TLR5 was expressed in tumor cells of all patients with gastric cancer, even though TLR4 expression was also detected in a considerable number of patients (24). Although TLR5 was expressed and responded to $H$. pylori flagellin in some gastric 
cancer cells (25), it is unknown whether and how TLR5 is involved in promoting the proliferation of gastric cancer cells. In this study, we examined the expression and function of TLR5 in various gastric cancer cells (SNU638, SNU601, SNU216, and AGS) and assessed whether bacterial flagellin enhances the growth of gastric adenocarcinoma.

\section{Materials and methods}

Cell culture and reagents. Gastric epithelial cell lines, SNU638, SNU601, SNU216, and AGS (human origin), were purchased from the Korean Cell Line Bank (KCLB, Seoul, Korea). The cells were incubated in RPMI medium including $10 \%$ fetal bovine serum (FBS) and $1 \mathrm{X}$ penicillin/streptomycin at $37^{\circ} \mathrm{C}$ containing $5 \% \mathrm{CO}_{2}$. Flagellin (Salmonella typhimurium origin) was purchased from Invivogen (San Diego, CA, USA). The extracellular-signal regulated kinase (ERK) inhibitor, PD98059, was purchased from Selleck Chemicals (Houston, TX, USA). Isotype IgG and anti-TLR5 antibody were purchased from Imgenex (San Diego, CA, USA).

RNA extraction and reverse transcription-polymerase chain reaction ( $R T-P C R)$. Total RNA was extracted from each cell using easy-BLUE (Intron Biotechnology, Daejeon, Korea). Total RNA $(1 \mu \mathrm{g})$ was reverse transcribed into cDNA and PCR was performed using the Power cDNA Synthesis kit (Intron Biotechnology) and the One-step RT-PCR AccuPower ${ }^{\circledR}$ HotStart PCR PreMix (Bioneer, Daejeon, Korea). The following primer sets were used: human TLR5, F: 5'-TGGGGGAACTT TACAGTTCG-3' and R:5'-CTGGGATTCTCTGAAGGGG-3'; human GAPDH, F: 5'-CGGAGTCAACGGATTTGGTC-3' and R: 5'-AGCCTTCTCCATGGTGGTGA-3'.

Enzyme-linked immunosorbent assay (ELISA). The cells were treated with the indicated doses of flagellin for $24 \mathrm{~h}$ and the culture supernatant was collected. The concentration of IL-8 in culture supernatants was determined by a commercial ELISA kit (R\&D Systems, Minneapolis, MN, USA).

Western blot analysis. The cells were lysed in a buffer containing $1 \%$ Nonidet P-40 supplemented with a complete protease inhibitor cocktail (Roche, Mannheim, Germany) and $2 \mathrm{mM}$ dithiothreitol. Lysates were resolved by $10 \%$ SDS-PAGE, transferred to polyvinylidene fluoride (PVDF) membrane, and immunoblotted with primary antibodies against regular and phopho-IкB- $\alpha$, p38, ERK, JNK, (Cell Signaling, Beverly, MA, USA). After immunoblotting with secondary antibodies, proteins were detected with an enhanced chemiluminescence (ECL) reagent (Intron Biotechnology).

Cell counting kit-8 (CCK-8) cell proliferation assay. The cells were treated with the indicated doses of flagellin for 3-4 days. Cell proliferation was determined by CCK-8 (Dojindo, Tokyo, Japan).

Statistical analysis. The differences between the mean values among the different groups were statistically assessed, and the values were expressed as the means $\pm \mathrm{SD}$. All of the statistical calculations were carried out using Microsoft Excel. Values of $\mathrm{P}<0.05$ were considered significant.

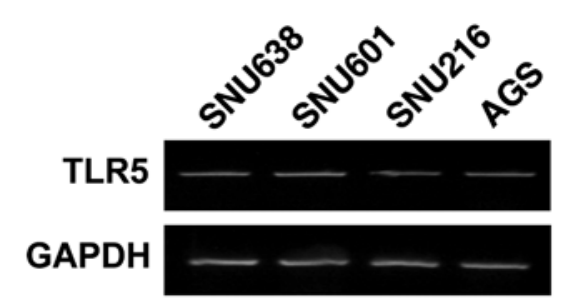

Figure 1. Gene expression of TLR5 in gastric cancer cells. RT-PCR was performed to examine TLR5 expression in four gastric cancer cell lines, SNU638, SNU601, SNU216, and AGS.

\section{Results}

Gene expression of TLR5 in gastric cancer cells. The gene expression of TLR5 was examined in the gastric cancer cell lines, SNU638, SNU601, SNU216, and AGS by RT-PCR. TLR5 mRNA was detectable in all tested cell lines and the expression level was similar between the cell lines (Fig. 1).

Flagellin leads to IL-8 production and NF- $\kappa B$ activation in gastric cancer cells. To determine whether TLR5 is functional in gastric cancer cells, the cells were treated with various doses of flagellin for $24 \mathrm{~h}$ and IL-8 production and NF- $\kappa B$ activation were examined. A large amount of IL-8 was produced in intact SNU638 cells, which was dose-dependently increased by flagellin (Fig. 2A). Likewise, treatment of flagellin increased IL-8 production in SNU601, SNU216, and AGS cells in a dose-dependent manner (Fig. 2B and C). However, LPS (a TLR4 agonist) did not affect IL-8 production in all cell lines (Fig. 2A and data not shown). Because the production of proinflammatory cytokines and chemokines is regulated by NF- $\kappa \mathrm{B}$, we examined the degradation and phosphorylation of IкB- $\alpha$ using Western blot analysis. Results showed that flagellin led to the phosphorylation of IкB- $\alpha$ 15 min after treatment in SNU638 and SNU601 cells (Fig. 2D and $\mathrm{E}$ ). The degradation of IкB- $\alpha$ by flagellin was also shown 30 (in SNU638) or $60 \mathrm{~min}$ (in SNU601) after treatment (Fig. 2D and E). These findings indicate that TLR5 responds to its ligands and may play a role in cellular signaling in gastric adenocarcinoma.

Flagellin augments the growth of gastric cancer cells. It was examined whether flagellin affects the growth of gastric cancer cells using CCK-8. As shown in Fig. 3A, treatment with flagellin, but not LPS, enhanced the proliferation of SNU638 cells. Similarly, the growth of SNU601, SNU216, and AGS cells was also augmented by flagellin (Fig. 3B). When TLR5 was blocked by its antibody, flagellin did not enhance the proliferation of SNU638 cells (Fig. 3C). These findings suggest that TLR5 may be involved in regulating the growth of gastric adenocarcinoma.

Flagellin leads to phosphorylation of ERK in SNU638 cells. Mitogen-activated protein kinases (MAPKs) are known to mediate multi-cellular processes such as proliferation, differentiation, migration, and apoptosis $(26,27)$. Western blot analysis was performed to determine whether flagellin induces the activation of MAPKs in gastric cancer cells. The results demonstrate that TLR5 stimulation with flagellin 


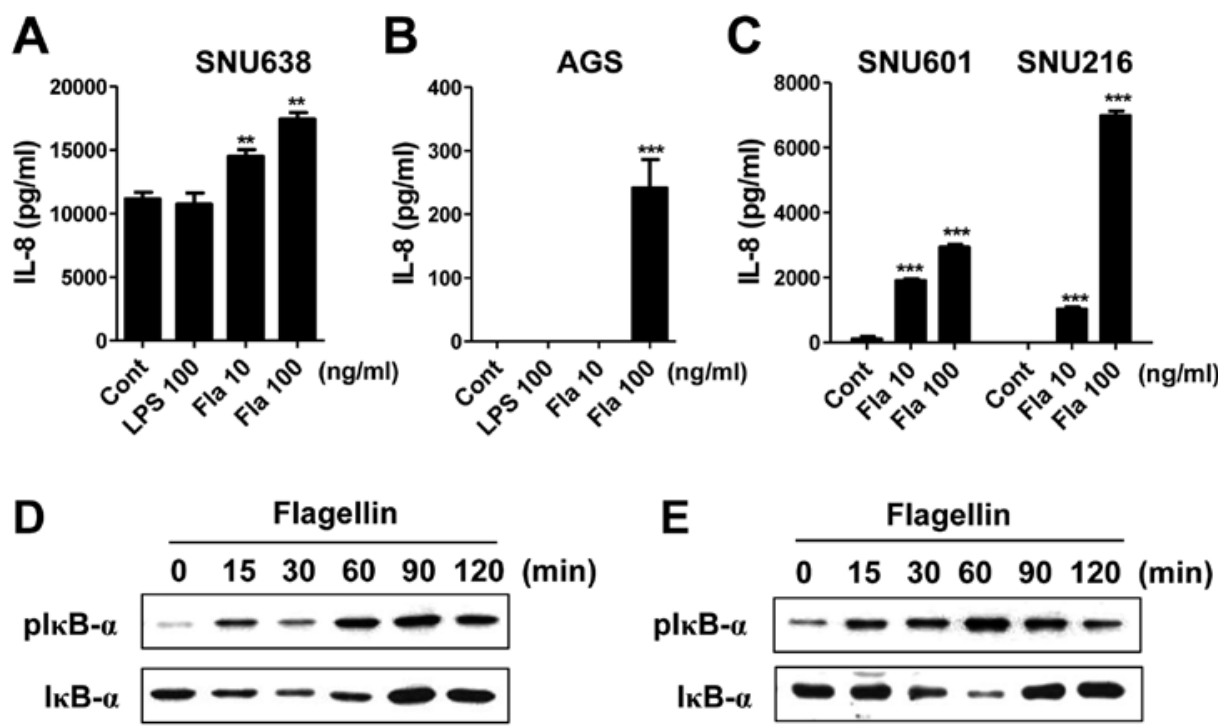

Figure 2. IL-8 production and NF-kB activation in gastric cancer cells. The cells were treated with the indicated doses of flagellin or LPS for $24 \mathrm{~h}$ and IL-8 production was measured in the culture supernatant (A-C). SNU638 (D) and SNU601 (E) cells were treated with flagellin (100 ng/ml) and protein was extracted at the indicated time points. Immunoblotting was performed using antibodies for the regular and phosphorylated form of I $\mathrm{B} B-\alpha(\mathrm{D}$ and $\mathrm{E}) .{ }^{* * *} \mathrm{P}<0.01,{ }^{* * * *} \mathrm{P}<0.001$.

A

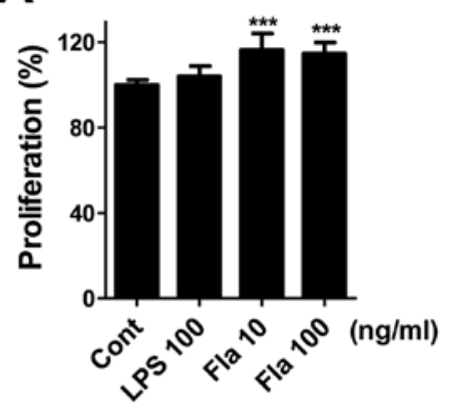

B
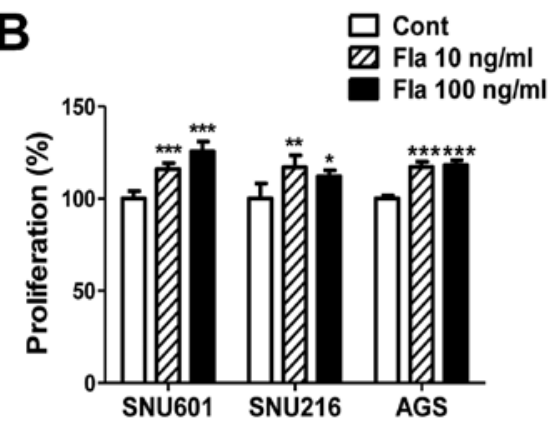

C

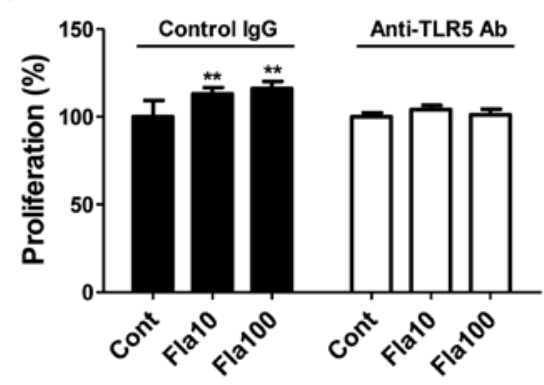

Figure 3. Cell proliferation assay. The cells $\left(2 \times 10^{3}\right.$ cells/well in a 96 -well plate) were incubated with indicated doses of flagellin or LPS for 3-4 days and cell proliferation was examined by CCK-8 (A-C). Anti-TLR5 Ab and isotype control $\mathrm{IgG}$ were pretreated $2 \mathrm{~h}$ before flagellin treatment $(\mathrm{C})$. ${ }^{*} \mathrm{P}<0.05,{ }^{* *} \mathrm{P}<0.01,{ }^{* * *} \mathrm{P}<0.001$.

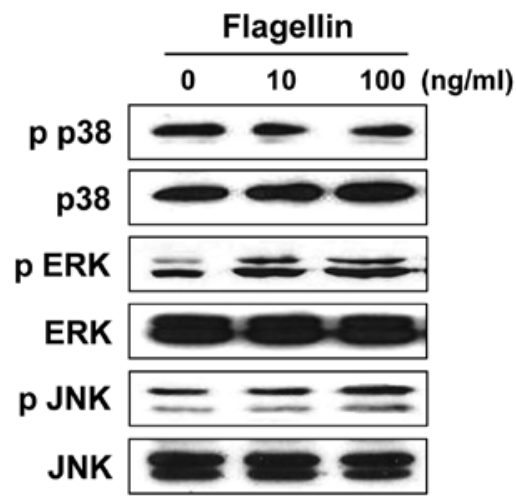

Figure 4. Activation of p38, ERK, and JNK in SNU638 cells. SNU638 cells $\left(1 \times 10^{6}\right.$ cells $/ 35-\mathrm{mm}$ dish) were treated with indicated doses of flagellin for $24 \mathrm{~h}$ and protein was extracted. Immunoblotting was performed using antibodies for the regular and phosphorylated forms of p38, ERK, and JNK.

leads to phosphorylation of ERK, but not of p38 and JNK, in SNU638 cells (Fig. 4).

ERK is critical for the proliferation of gastric cancer cells enhanced by flagellin. Finally, we examined the role of ERK on the proliferation of gastric cancer cells by flagellin using an inhibitor assay. Treatment with the ERK inhibitor, PD98059, restored the flagellin-enhanced proliferating ability of SNU638 cells (Fig. 5), suggesting that ERK may play an important role in the proliferation of gastric cancer cells.

\section{Discussion}

TLR signaling is much like a double-edged sword in tumor development and progression. TLRs are involved in not only tumor progression by promoting cell proliferation, migration, invasion, and angiogenesis, but also in tumor suppression by inducing cell death or immune activation $(4,28)$, which are critically dependent on the tumor origin or the TLR type. 


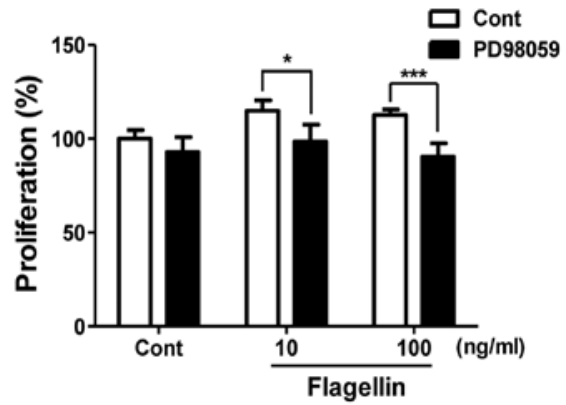

Figure 5. ERK inhibitor assay. SNU638 cells were treated with the ERK inhibitor, PD98059, for $2 \mathrm{~h}$ before flagellin treatment. The cell proliferation assay was performed as described in Fig. $3 .{ }^{*} \mathrm{P}<0.05,{ }^{* * * *} \mathrm{P}<0.001$.

The promotion of cell proliferation by microbial molecules via TLR signaling has been widely studied. Peptidoglycan, lipoteichoic acid, synthetic lipoprotein, and flagellin have been repoted to increase epithelial cell growth via TLR2 or TLR5 (15). Listeria monocytogenes was shown to induce $\mathrm{NF}-\kappa \mathrm{B}$ and MAPK activation and to promote the growth of $\mathrm{H} 22$ cells (a hepatocarcinoma cell line) via TLR2 signaling (22). TLR4 and TLR9 have been associated with cell proliferation of prostate epithelial cells through unknown mechanisms (29). These studies suggest that various TLRs are involved in tumor growth through distinct mechanisms.

In gastric cancers, TLR2 and TLR4 are known to promote cell proliferation $(23,30)$. However, a recent clinical study revealed that TLR5 was strongly expressed in tumor cells of all examined patients $(n=22)$ with gastric carcinoma (24), which drove us to examine the role of TLR5 on the growth of gastric cancer. In this study, we showed that the TLR5 gene was functionally expressed in all gastric cancer cell lines tested, which was supported by the enhanced IL-8 production and NF- $\kappa \mathrm{B}$ activation induced by a TLR5 agonist, flagellin. However, LPS did not increase IL-8 production in any of the cell lines examined, consistent with previous studies. Smith et al (25) revealed that two gastric epithelial cell lines, AGS and MKN45, express TLR4, but not MD-2 (a TLR4 co-receptor), which is essential for TLR4 function. A transfection assay also showed that LPS did not induce $\mathrm{NF}-\kappa \mathrm{B}$ activation in MKN45 cells, suggesting that TLR4 is non-functional in these cells (25). Only TLR2 and TLR5, but not TLR4, were required for $H$. pylori-induced $\mathrm{NF}-\kappa \mathrm{B}$ activation in MKN45 cells (25). In addition, it was clarified that $E$. coli LPS produced very low levels of IL-8 in intact gastric epithelial cells (MKN28 and MKN45 cells), which was due to a low level expression of TLR4 $(30,31)$. However, H. pylori LPS up-regulated TLR4 expression in those cells via TLR2 signaling, which enhanced the $E$. coli LPS-induced IL-8 production (30). These findings suggest that TLR4 may be functional after stimulation increasing its expression in gastric epithelial cells, although it exhibits low activity under intact conditions.

In this study, flagellin enhanced the proliferation of all gastric cell lines tested, whereas LPS did not enhance the proliferation of SNU638cells. This finding is in discordance with a study by Chochi et al (23) showing that treatment of LPS from $E$. coli and $H$. pylori up-regulated the proliferation of gastric cancer cell lines including MKN45, which was mediated by TLR4. Although it can be due to differences in the experimental protocol and the conditions, the exact reason for the discrepancy remains to be elucidated.

ERK is associated with tumor proliferation and malignancy in gastric cancers. H. pylori LPS induced the proliferation of gastric cancer cells via the MEK1/2-ERK1/2 pathway (30). TGF- $\beta$ also promoted invasion and metastasis of gastric cancer cells via the ERK and JNK signaling pathway (32). In addition, Mycoplasma hyorhinis infection promoted tumor cell migration, invasion, and metastasis, which was possibly associated with the enhanced ERK phosphorylation (33). In this study, flagellin enhanced ERK phosphorylation in SNU638 cells and the ERK inhibitor, PD98059, restored the proliferating ability of SNU638 cells enhanced by flagellin, suggesting that flagellin may promote cell proliferation through an ERK-dependent pathway.

In conclusion, our study revealed that TLR5 activation enhances the proliferation of gastric cancer cells through the ERK signaling pathway. Further studies examining TLR5 functions, such as angiogenesis, metastasis, and immune modulation in gastric cancers and its physiological role using an in vivo tumor model are warranted.

\section{Acknowledgements}

This study was supported by a program for general research from the National Research Foundation of Korea (No. 20100015722).

\section{References}

1. Akira S, Uematsu S and Takeuchi O: Pathogen recognition and innate immunity. Cell 124: 783-801, 2006.

2. Erridge C: Endogenous ligands of TLR2 and TLR4: agonists or assistants? J Leukoc Biol 87: 989-999, 2010.

3. Lee MS and Kim YJ: Signaling pathways downstream of pattern-recognition receptors and their cross talk. Annu Rev Biochem 76: 447-480, 2007.

4. Rakoff-Nahoum S and Medzhitov R: Toll-like receptors and cancer. Nat Rev Cancer 9: 57-63, 2009.

5. Li X, Jiang S and Tapping RI: Toll-like receptor signaling in cell proliferation and survival. Cytokine 49: 1-9, 2010.

6. Huang B, Zhao J, Li H, et al: Toll-like receptors on tumor cells facilitate evasion of immune surveillance. Cancer Res 65: 5009-5014, 2005.

7. Kim S, Takahashi H, Lin WW, et al: Carcinoma-produced factors activate myeloid cells through TLR2 to stimulate metastasis. Nature 457: 102-106, 2009.

8. Harmey JH, Bucana CD, Lu W, et al: Lipopolysaccharideinduced metastatic growth is associated with increased angiogenesis, vascular permeability and tumor cell invasion. Int J Cancer 101: 415-422, 2002.

9. Ilvesaro JM, Merrell MA, Swain TM, et al: Toll like receptor-9 agonists stimulate prostate cancer invasion in vitro. Prostate 67: 774-781, 2007.

10. Merrell MA, Ilvesaro JM, Lehtonen N, et al: Toll-like receptor 9 agonists promote cellular invasion by increasing matrix metalloproteinase activity. Mol Cancer Res 4: 437-447, 2006.

11. Kelly MG, Alvero AB, Chen R, et al: TLR-4 signaling promotes tumor growth and paclitaxel chemoresistance in ovarian cancer. Cancer Res 66: 3859-3868, 2006.

12. Wang JH, Manning BJ, Wu QD, Blankson S, Bouchier-Hayes D and Redmond HP: Endotoxin/lipopolysaccharide activates NF-kappa B and enhances tumor cell adhesion and invasion through a beta 1 integrin-dependent mechanism. J Immunol 170: 795-804, 2003.

13. Yuan ZQ, Feldman RI, Sussman GE, Coppola D, Nicosia SV and Cheng JQ: AKT2 inhibition of cisplatin-induced JNK/p38 and Bax activation by phosphorylation of ASK1: implication of AKT2 in chemoresistance. J Biol Chem 278: 23432-23440, 2003. 
14. Hua D, Liu MY, Cheng ZD, et al: Small interfering RNA-directed targeting of Toll-like receptor 4 inhibits human prostate cancer cell invasion, survival, and tumorigenicity. Mol Immunol 46: 2876-2884, 2009.

15. Shaykhiev R, Behr J and Bals R: Microbial patterns signaling via Toll-like receptors 2 and 5 contribute to epithelial repair, growth and survival. PLoS One 3: e1393, 2008.

16. Xie W, Wang Y, Huang Y, Yang H, Wang J and Hu Z: Toll-like receptor 2 mediates invasion via activating NF-kappaB in MDA-MB-231 breast cancer cells. Biochem Biophys Res Commun 379: 1027-1032, 2009.

17. Danaei G, Vander Hoorn S, Lopez AD, Murray CJ and Ezzati M: Causes of cancer in the world: comparative risk assessment of nine behavioural and environmental risk factors. Lancet 366 : 1784-1793, 2005.

18. Parkin DM, Bray F, Ferlay J and Pisani P: Global cancer statistics, 2002. CA Cancer J Clin 55: 74-108, 2005.

19. Herrera V and Parsonnet J: Helicobacter pylori and gastric adenocarcinoma. Clin Microbiol Infect 15: 971-976, 2009.

20. Santini D, Angeletti S, Ruzzo A, et al: Toll-like receptor 4 Asp299Gly and Thr399Ile polymorphisms in gastric cancer of intestinal and diffuse histotypes. Clin Exp Immunol 154: 360-364, 2008

21. El-Omar EM, Ng MT and Hold GL: Polymorphisms in Toll-like receptor genes and risk of cancer. Oncogene 27: 244-252, 2008.

22. Huang B, Zhao J, Shen S, et al: Listeria monocytogenes promotes tumor growth via tumor cell toll-like receptor 2 signaling. Cancer Res 67: 4346-4352, 2007.

23. Chochi K, Ichikura T, Kinoshita M, et al: Helicobacter pylori augments growth of gastric cancers via the lipopolysaccharidetoll-like receptor 4 pathway whereas its lipopolysaccharide attenuates antitumor activities of human mononuclear cells. Clin Cancer Res 14: 2909-2917, 2008.

24. Schmausser B, Andrulis M, Endrich S, Muller-Hermelink HK and Eck M: Toll-like receptors TLR4, TLR5 and TLR9 on gastric carcinoma cells: an implication for interaction with Helicobacter pylori. Int J Med Microbiol 295: 179-185, 2005
25. Smith MF, Jr., Mitchell A, Li G, et al: Toll-like receptor (TLR) 2 and TLR5, but not TLR4, are required for Helicobacter pyloriinduced NF-kappa B activation and chemokine expression by epithelial cells. J Biol Chem 278: 32552-32560, 2003.

26. Dhillon AS, Hagan S, Rath O and Kolch W: MAP kinase signalling pathways in cancer. Oncogene 26: 3279-3290, 2007.

27. Kim EK and Choi EJ: Pathological roles of MAPK signaling pathways in human diseases. Biochim Biophys Acta 1802: 396-405.

28. Wolska A, Lech-Maranda E and Robak T: Toll-like receptors and their role in carcinogenesis and anti-tumor treatment. Cell Mol Biol Lett 14: 248-272, 2009.

29. Kundu SD, Lee C, Billips BK, et al: The toll-like receptor pathway: a novel mechanism of infection-induced carcinogenesis of prostate epithelial cells. Prostate 68: 223-229, 2008

30. Yokota S, Okabayashi T, Rehli M, Fujii N and Amano K: Helicobacter pylori lipopolysaccharides upregulate toll-like receptor 4 expression and proliferation of gastric epithelial cells via the MEK1/2-ERK1/2 mitogen-activated protein kinase pathway. Infect Immun 78: 468-476, 2010.

31. Yokota S, Ohnishi T, Muroi M, Tanamoto K, Fujii N and Amano K: Highly-purified Helicobacter pylori LPS preparations induce weak inflammatory reactions and utilize Toll-like receptor 2 complex but not Toll-like receptor 4 complex. FEMS Immunol Med Microbiol 51: 140-148, 2007.

32. Fu H, Hu Z, Wen J, Wang K and Liu Y: TGF-beta promotes invasion and metastasis of gastric cancer cells by increasing fascin1 expression via ERK and JNK signal pathways. Acta Biochim Biophys Sin 41: 648-656, 2009.

33. Yang H, Qu L, Ma H, et al: Mycoplasma hyorhinis infection in gastric carcinoma and its effects on the malignant phenotypes of gastric cancer cells. BMC Gastroenterol 10: 132, 2010. 